\title{
The Sensor Web: Advanced Technology for Situational Awareness
}

\author{
Kevin A. Delin \\ SensorWare Systems, Inc. \\ and \\ Edward Small \\ Captain, Sacramento Metropolitan Fire District; \\ FEMA Urban Search and Rescue Team, CA Task Force 7
}

\begin{abstract}
The Sensor Web is a wireless sensor infrastructure for collecting, synthesizing, distributing, viewing, and reacting to information. While the Sensor Web is well suited to collecting data in hazardous or inhospitable locations, the system has considerable value when situational monitoring must be accomplished over an extended area. The Sensor Web can provide situational awareness in many types of applications including disaster preparedness and recovery, emergency response, health and safety, infrastructure protection, homeland security and national defense. Uses of the Sensor Web are virtually limitless since different types of sensors can be connected to the system for different functionalities. Here, the primary application focus will be that related to emergency services and rescue operations. Actual deployments will also be discussed.
\end{abstract}

\section{INTRODUCTION}

The need for situational awareness in the dynamic environment of emergency and rescue operations is well understood. Data must be continually collected, analyzed, assimilated, and disseminated to both local operational personnel and remote commanders. The basic principles of "Facts, Probabilities, Own Situation, Decision, and Plan of Operation" for fire and rescue strategies are just as relevant today as they were when originally described in 1953[1]. Simply stated, situational awareness informs decision making and decreases reaction time to changing conditions, even allowing for anticipation of events in certain instances. 
Failure to effectively collect, synthesize, and distribute facts to personnel involved at all levels of a field operation will result in service delays, or worse, death. Emergency services personnel cannot begin operations without having the ability to monitor for hazards and account for personnel. Because emergency and rescue operations are labor intensive, however, continuous and effective monitoring for hazardous conditions often becomes less of a priority, or disappears entirely. It is therefore of critical importance to find a technological means to generate situational awareness for those personnel working in the hazardous area, both as a means to speed the course of the operation and to protect the personnel from danger.

Here, one such new piece of equipment, the Sensor Web, is examined. This technology can aid and substitute for human efforts in understanding the changing, and often chaotic, conditions during emergency service operations. First, the Sensor Web technology will be briefly described. Then, a series of representative field applications, including actual operations, will be given that illustrate the unique capabilities of the Sensor Web as applied to emergency services. Finally, future directions of the technology are considered.

\section{SENSOR WEB TECHNOLOGY}

The Sensor Web is an embedded, intelligent infrastructure for sensors. Physically, it consists of spatially distributed sensor/actuator platforms (called "pods") that wirelessly communicate with one another (see Fig.1). Originally developed at NASA for planetary exploration of unknown environments, the Sensor Web is also well-suited for providing situational awareness in the chaotic and unpredictable environments associated with emergency and rescue operations. Despite its sophistication, such a system would cost no more than traditional, less capable wireless solutions and could actually reduce total operational costs by providing continual, automated in-field analysis thereby freeing up rescue personnel for other, more demanding, tasks.

\subsection{Sensor Web Protocols}

The wireless communication between pods should be thought of as an information bus, in the same way that buses connect the individual components (hard drive, optical drive, memory, logic units, etc.) of a computer. Consequently, while the individual pods are certainly networked with each other, the Sensor Web is not, in and of itself, a network but rather a spatially distributed macro-instrument[2]. The distinction is crucial: a network consists of components that route information along communication paths to specific destination points, while a macro-instrument consists of components that share information with each and every other piece at all times without any intervening routing. The Sensor Web's applicability to situational awareness, in fact, derives from having its sensor measurements taken, distributed, and interpreted collectively over this unique, massively redundant, communication architecture.

Stated another way, every pod pushes its data out onto the Sensor Web in an omni-directional manner. There is never a purposeful routing of data towards a specific pod or a special portal or gateway. In this way, every pod is made aware of conditions throughout the entire Sensor 


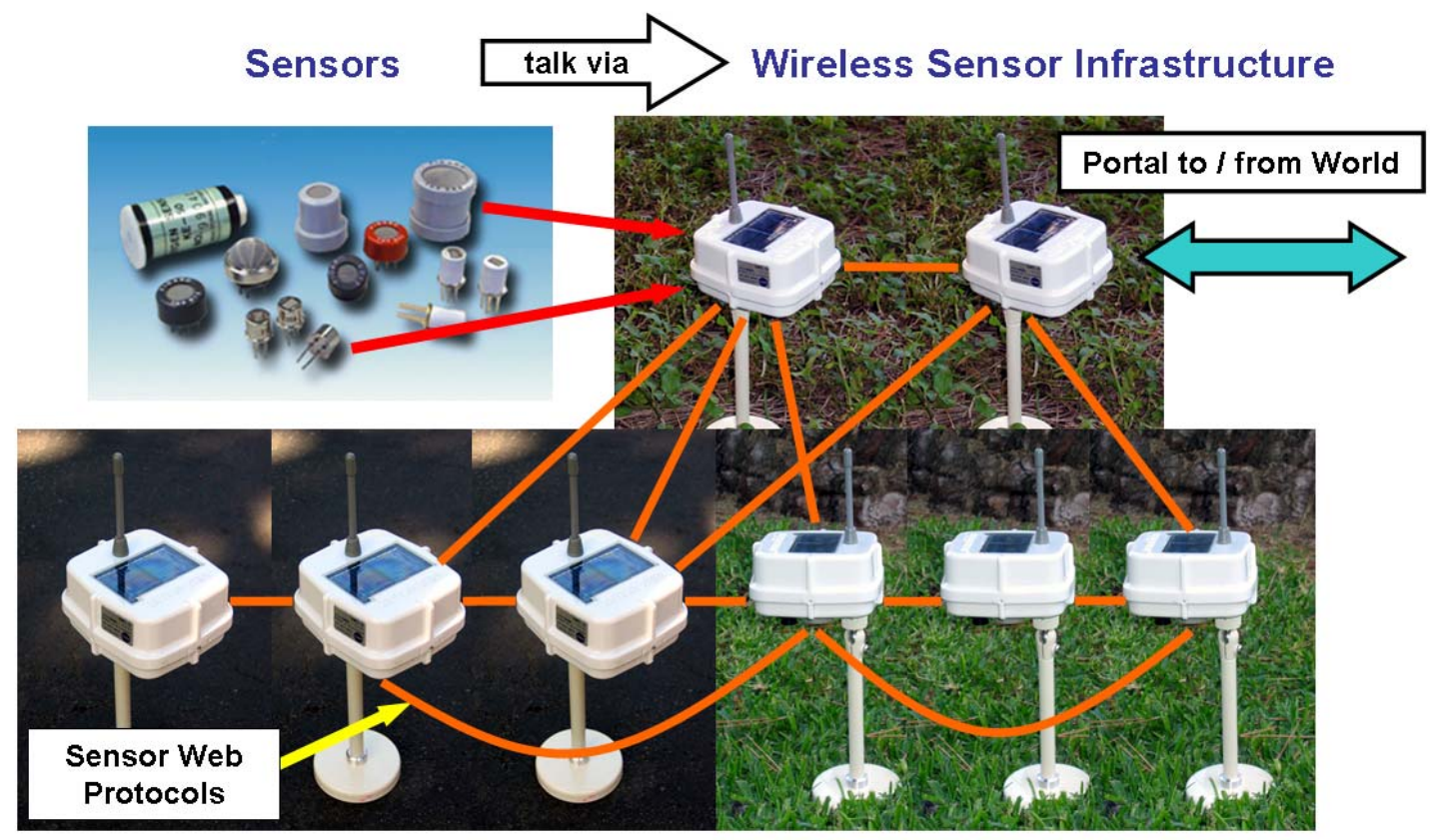

Figure 1: Schematic representation of a Sensor Web. Sensors are connected to the pods (white boxes). The pods communicate wirelessly to form an amorphous network where all pods are equivalent and any pod can be a portal to the outside world.

Web during each measurement cycle. In fact, while the computation hardware in a pod can be quite sophisticated, it is the sharing of information among the pods that gives the Sensor Web its macro-intelligence. This is similar to how perception is created in the brain from a complex, interacting set of neurons that share electro-chemical signals[3] rather than from individual intelligence at each neuron.

The Sensor Web communication architecture of data sharing is distinct from both hub-andspoke and mesh network types. In hub-and-spoke networks, individual spoke nodes can be synchronized to the hub but information must always be routed through the hub to get to other points. Mesh networks are typically based on asynchronous Internet-like (TCP/IP) protocols and require information routing as well. In marked contrast to these two network types, the Sensor Web communication architecture, by design, is synchronous (all measurements across the system are taken at the same time) and requires no routing.

The Sensor Web communication protocols are simple and robust. Each measurement cycle begins with the pods taking in sensor data. After a measurement is taken, each individual pod in the system broadcasts its information (data it has taken or received from others) in an omni-directional manner to all pods in communication range. Each pod then processes and analyzes the information it has received and the cycle repeats. In this way, information is hopped pod-to-pod and spread throughout the entire Sensor Web. The entire system becomes a coordinated whole by possessing this internal, continuous data stream, drawing knowledge from it, and reacting to that knowledge. 


\subsection{Sensor Web Pods}

A key feature of the Sensor Web is that its component parts, the sensor platforms or pods, are all alike (see Fig. 2). In general, they only differ by the sensors attached to them. A single pod, known as the mother, holds the single, system-wide clock that will synchronize all pods. The mother, however, holds no special hardware; indeed any pod may be designated as the mother simply by labeling it as such. Unlike hub-and-spoke and mesh networks, the Sensor Web is a truly amorphous network with no central point and no specially designated portal or gateways.

Each Sensor Web pod consists of five basic modules:

1. The radio: Although any radio frequency can be chosen, the $900 \mathrm{MHz}$ license-free Industrial, Science and Medical (ISM) band has been used in manufactured Sensor Web systems to date. This frequency requires no licensing of end-users and does not compete with the more common frequencies found at emergency sites (minimizing jamming). In addition, radios operating at this frequency do not require line-of-sight communication (even going through concrete walls) and have an upper range of about $200 \mathrm{~m}$ (compliant with government power regulations). Because each pod will
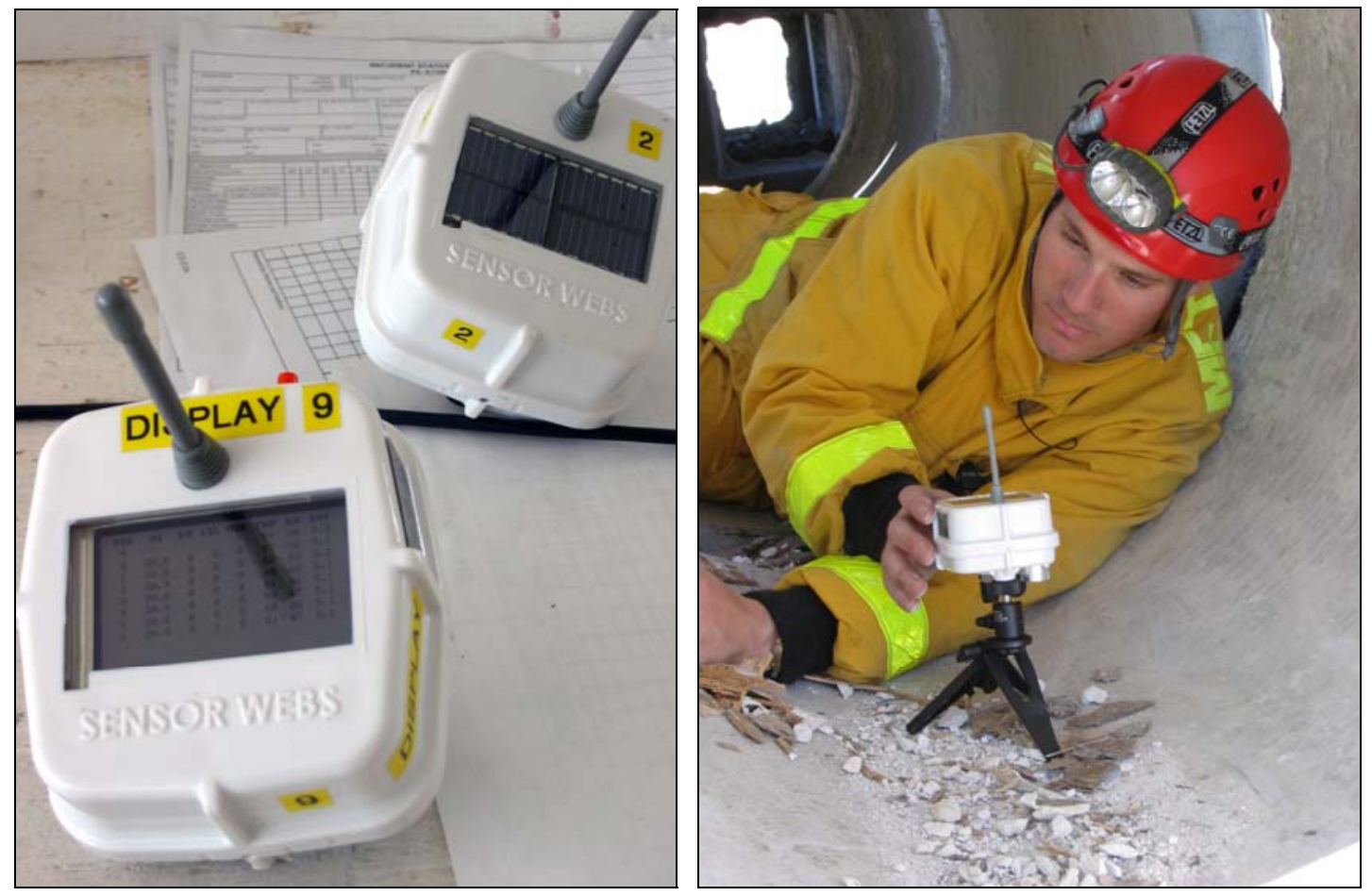

Figure 2: Sensor Web pods. Left: A standard pod is in the background and shows both the 900 MHz antenna and a solar panel to harvest additional energy for the pod's rechargeable batteries. In the foreground is a special display pod where a flat panel display has replaced the solar panel and reveals the conditions at other pods. This allows mobile personnel to monitor the Sensor Web without having to access a computer. Labels have been attached to the pods to clearly mark their software-assigned identification. Right: A responder deploys a Sensor Web pod. Pods can be mounted using a variety of hardware including stands, magnetic bases, and spikes. 
essentially function as a repeater and retransmit data it receives from other pods, the effective radio range is extended far beyond the limits imposed by the specifications and regulations associated with a single radio.

2. The microprocessor: This component contains the system's protocols, communicates with the attached sensors, and carries out data analysis as needed.

3. The power system: The combination of solar panels, rechargeable batteries and micropower electronic design have kept Sensor Web pods operating in the field for years without requiring maintenance.

4. The pod packaging: The package is lightweight, durable, inexpensive, and sealed against such elements as rain, standing water, water sprays, dust storms, and caustic chemicals. In addition, it provides for easy and rapid mounting.

5. The sensor suite: This module is completely determined by the specific application. It is the ability to accommodate a wide range of sensor types that makes the Sensor Web so versatile. For the types of operations discussed here, typical sensors include those for monitoring gases and environmental conditions such as air temperature and humidity.

\subsection{Sensor Web Properties}

As just described, the Sensor Web is a distributed, macro-instrument based on its unique protocols that allow for data sharing via non-routed, synchronous inter-pod communication. These characteristics create valuable Sensor Web properties when the technology is applied to emergency and rescue operations (see Fig. 3).

1. The redundant, non-routed, data sharing allows for any pod to be a portal to the outside world. Since every piece of the Sensor Web contains all the same information, anyone with access to a single pod will be provided with the same situational awareness picture. This means that the first-responder at the rescue scene looking at the flat panel display of a pod, the incident commander directing operations who has plugged his laptop computer into another pod, and remote government personnel examining the information of a third pod being sent out over the Internet will, simultaneously, have a common, unified picture of operations.

2. A single, system-wide clock provides for an immediate synchronous snapshot that can be intuitively understood by field personnel. Because the Sensor Web is synchronous, all measurements are taken at the same time and, in essence, become a pixel in the overall picture taken by the Sensor Web. This picture is an immediate integration and valuable for in-field personnel to understand plume motion, for example, without the need for time-consuming post-hoc analysis. Moreover, when combined with the massively redundant, non-routed communication paths, this snapshot is known to all pods in the Sensor Web which allows for anticipatory warnings and alarms. In this way, field personnel are alerted to dangerous conditions 


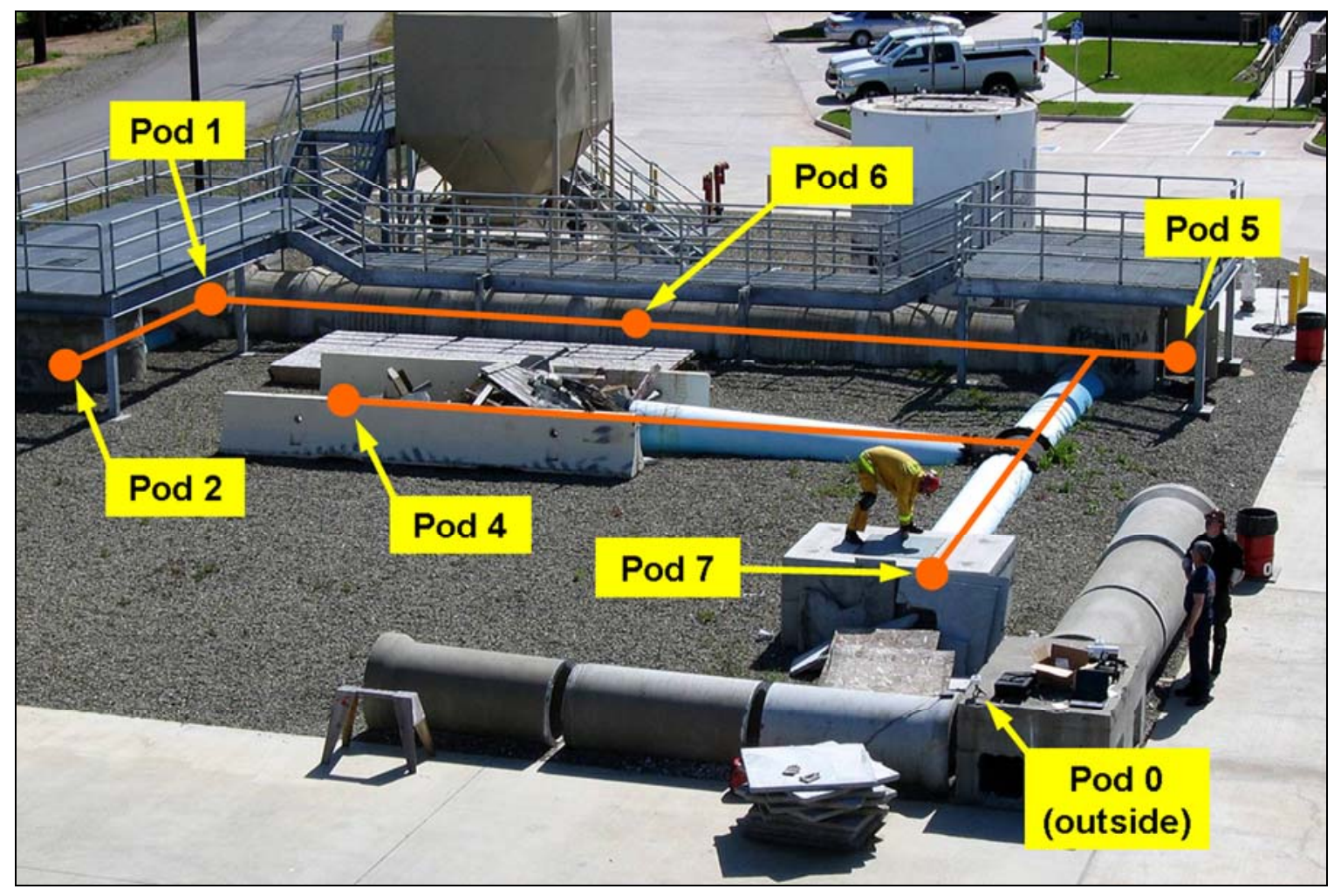

Figure 3: A confined space training facility for Urban Search and Rescue. The Sensor Web system was deployed inside the structure as indicated schematically by the dots. The size of the structure is indicated by the size of the firefighter on top of the structure near pod 7 . Lines connecting dots indicate physical paths, not pod connectivity. When deployed, the pods were able to wirelessly communicate through the concrete barriers; for example, pod 4 was in direct communication with pods 1,2 , and 6 . When a chain saw was intentionally left running between pods 5 and 6 , personnel were able to observe the migration of carbon monoxide through the structure with one front towards pods 6, 1, and 2 and a secondary front towards pod 7 . All pods in the system went into alarm together when the gas first reached threshold levels at pod 6.

anywhere in the work zone immediately by the Sensor Web rather than by a remote incident commander.

3. Synchronous system behavior reduces latency between data taking and data reporting. Each pod has the same situational awareness picture at the end of every measurement cycle. Because each pod can act as a portal into the system, this implies that all endusers are kept up-to-date on every measurement cycle.

4. The massively redundant, non-routed communication paths provide for a highly robust structure with no central point of system failure. Since all pods are potential portals, the loss of any particular pod does not effect the entire operation. Since any pod can be designated as the single, system-wide clock, the worse case scenario of a damaged mother pod is rapidly recoverable simply by assigning (via a software label) the designation of "mother" to another pod. This worse case failure can even be corrected automatically without human intervention if, after a predetermined amount 
of time without receiving signal from the mother, the pod with the current lowest serial number in the Sensor Web promotes itself to mother.

5. The redundant, non-routed, data sharing allows for recoverable single point sensor failure. Because every pod contains a microprocessor and knows all sensor measurements across the system at each measurement cycle, each pod can locally analyze global conditions across the entire Senor Web. It is therefore possible for the system to immediately evaluate a seemingly anomalous measurement against the background of neighboring measurements to determine, on a statistical basis, false positives or to distinguish between a merely worsening trend versus a true critical situation requiring evacuation. It is also possible for the system to "suggest," within the same measurement cycle, a missing sensor measurement by combining spatial interpolation of neighboring measurements with recent local measurement trends.

6. The massively redundant, non-routed communication paths allow the system to be easily and rapidly deployed. No special skill is required to set up a Sensor Web as all the pods are essentially the same (in contrast to other networking schemes which require special hardware gateways or router tables). As a result, once the mother pod is switched on to provide a clock for the system, the pods may be dispersed as dictated by the needs of the situation. Data taking is immediate and the pods may even be reshuffled on-the-fly and, as long as they stay within communication range, maintain the overall Sensor Web macro-instrument. This is particularly valuable if pods are assigned to specific rescue squads that are each moving independently through a building. Because emergency service operations do not allow the time to leisurely recall complex equipment function, the simplicity and speed of deployment may be among the Sensor Web’s most compelling and important features.

\section{SENSOR WEBS APPLIED TO FIELD OPERATIONS}

To date, there have been over 30 Sensor Web field deployments with systems spanning distances up to 6 miles and running continuously for over 3 years. The systems have been tested extensively in numerous, challenging environments from the remote ice slopes of Antarctica to the searing heat of the central New Mexico desert to the corrosive salt air of the Florida coast[4-6]. Real-time, streaming output of some of these systems may be viewed over the Internet using a variety of user interface displays[6]. Here, the Sensor Web capabilities as applied specifically to emergency and rescue operations will be examined. As will be shown, the Sensor Web's properties and physical robustness allow it to efficiently bring key environmental parameters together in a continuous operational picture and disseminate this picture to in-field and remote personnel.

A critical issue for any type of deployment is determining a pod's location. For extended, outdoor operations, this is most easily accomplished by using an external Global Positioning System (GPS) unit during deployment, noting pod placement coordinates, and putting these coordinates into the pod's memory as it is deployed. Pods can then share their individual coordinates with each other just as they do with sensor data. If pod power usage, size, and 
cost are not an issue, it is also possible to place a GPS unit inside each pod. Sometimes, however, GPS coordinates may not be a practical option because (a) pods may be shielded from strong GPS signals (as when placed inside a building), (b) building geometry provides a more transparent understanding of pod placement (e.g. "pod at the west end of the first floor corridor"), or (c) typical GPS resolution may not be accurate enough (as in the case of placing a pod against a wall and determining which room it is in). In such cases, simple hand-mapping by in-field personnel has been found to be effective and easily performed, even under rapid deployment circumstances. In addition, these hand-mapped "coordinates" typically provide rescue personnel with a clearer, intuitive picture of how the Sensor Web is deployed in the area. Pods can still autonomously perform spatial data analysis in these cases because a relative pod placement map can be formed by each pod through shared knowledge of every individual pod's nearest neighbors.

\subsection{Atmospheric Monitoring}

The predominant cause of death in a confined space incident is from a hazardous atmosphere. Typically, in a confined space or structural collapse operation, one highly competent person is dedicated to the position of "environmental officer". This person continuously monitors the atmosphere by way of a gas sensor with remote sampling capability, or by periodically requesting a reading from the entrant who is carrying a gas sensor, or both. This person also directs the forced ventilation efforts to enhance victim survivability and to maintain as tenable, and explosion-free, an atmosphere as possible.

There are several difficulties associated with the present technique. First, because all of the gases monitored have different vapor densities, they tend to stratify at different levels in the space, or may become trapped in dead spaces and fail to diffuse into the atmosphere. For this reason, atmospheric sampling must occur at 4 foot intervals, both vertically and horizontally. This significantly slows the progress of field personnel motion as they move into or even exit a confined space operation. Second, while the use of a sampling pump and tubing is a common method of obtaining remote gas samples within the confined space, there is the issue of the time it takes for the atmospheric sample to be drawn through the sample tubing. Since this may take up to $3 \mathrm{sec} / \mathrm{ft}$ of tubing and 50 foot sections of tubing are not uncommon, gas monitoring can require 1.5 to 2.5 minutes of delay per sample. Third, with the portable devices typically used by rescue personnel, evacuation of the space is often at the discretion of those working. Experience has shown that most in-field personnel consider an alarm to be an annoyance, and remain focused on completing their task; the atmosphere is only a "little hazardous". Finally, documentation of the atmospheric monitoring for most types of confined space operation is required by state/federal law, yet maintaining this documentation during the operation takes away from the actual emergency or rescue services.

Sensor Webs have been built and successfully used for monitoring confined space atmospheres. Here, the pods are equipped with the 4 gas sensors necessary for this application (e.g. oxygen, carbon monoxide, hydrogen sulfide, and explosive limits). The pods also contain sensors for air temperature and humidity. The measurement cycle for this Sensor Web is programmed for 30 seconds. These systems have been used for several years now in confined space operations and collapsed structure training exercises. It has been 

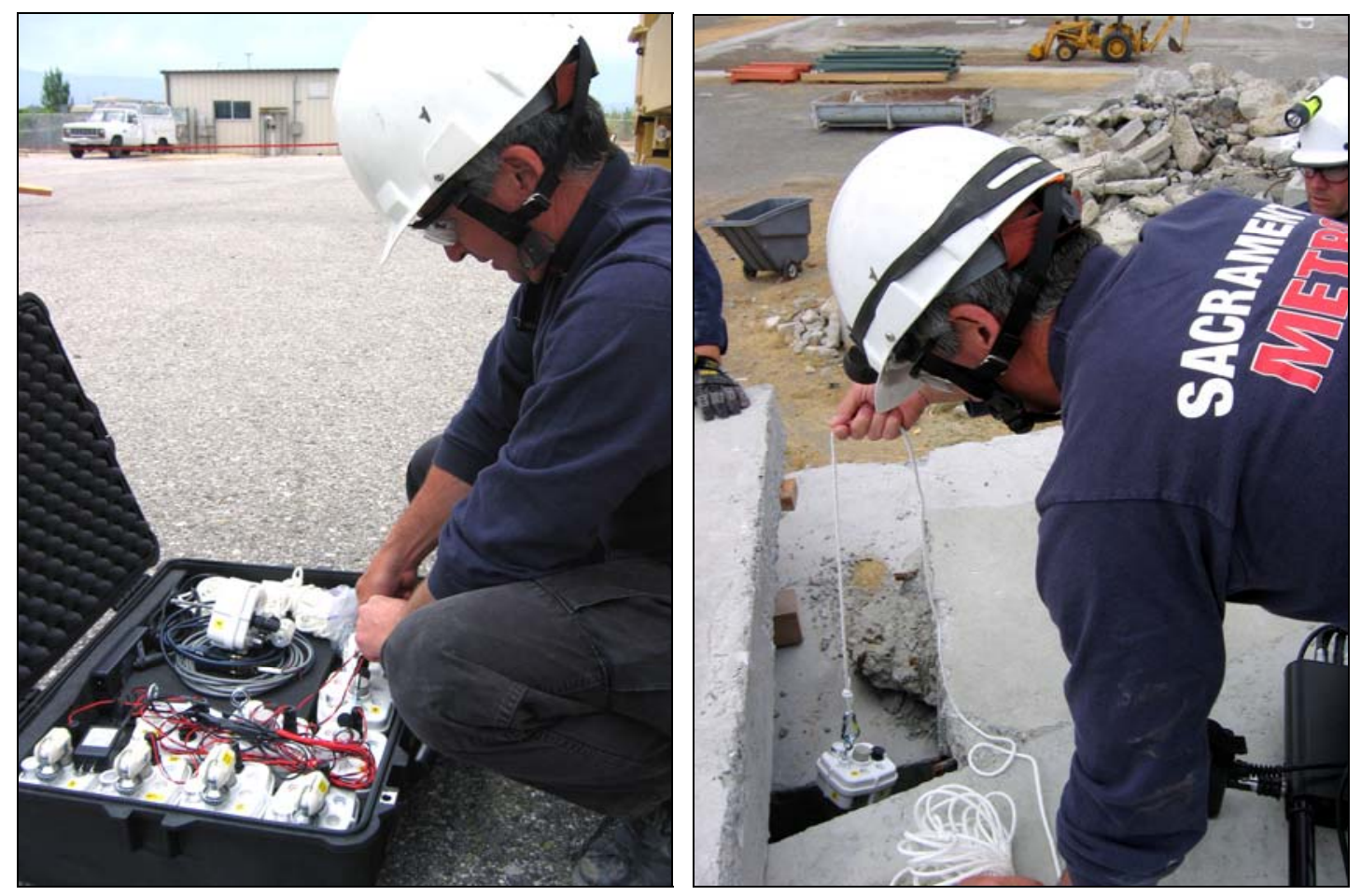

Figure 4: Sensor Web used for atmospheric monitoring. Left: A 10-pod Sensor Web system, including laptop and several varieties of pod mounting hardware fit compactly in a case, ready for rapid field deployment. Right: A pod being lowered into a confined space to determine atmospheric conditions. The pod will be left in this space for the duration of the operation allowing personnel to freely move into and out of the area.

found that the rescue personnel readily adapt to the new technology and have no difficulty infusing the technology into standard procedures (see Fig. 4).

Benefits of using the Sensor Web for atmospheric monitoring include:

1. Providing a permanent sensing infrastructure that frees the rescue personnel from having to take measurements. As a squad penetrates the confined space or collapsed structure, it deploys pods along the ingress path, effectively growing the Sensor Web. Once in place, the Sensor Web allows personnel to move freely into and out of the operations area. This is especially important during lengthy operations where squads will be replaced periodically. Moreover, since all pods would alarm when any pod detects a hazard, other responders working in other portions of the building will be aware if there is a gas leak that could affect them as well. As a result, responders will quickly know when to exit and how to modify egress paths by detecting remote atmospheric changes due to gas leaks.

2. Reducing the latency of obtaining measurements compared to drawing gas through a tube. Measurements are now available to the environmental officer at the sampling frequency (here, 30 seconds) throughout the entire field of operation. 
3. Providing the environmental officer and incident commanders with a full picture of atmospheric conditions without diverting the rescuer's attention from other crucial tasks (see Fig. 5). During an actual collapsed structure training operation, the Sensor Web revealed trends of oxygen displacement from the expired carbon dioxide of rescuers in confined areas, as well as increases in temperature and humidity from rescuers and equipment. This allowed the incident commander to move ventilation fans from other parts of the operation into the affected confined areas to allow rescuers to proceed without stopping. While the Sensor Web system provides warning of imminent hazardous conditions, just like the single station gas detectors, the greater value may be in its ability to display trends in environmental conditions and disseminate that information to commanders with authority to act.

4. Providing an accurate and immediate record of conditions recorded every 30 seconds by the Sensor Web and output to a laptop connected to the mother pod.

\subsection{Structural Integrity Monitoring}

The dangers present in and around structures compromised by natural or man-made disasters are rarely static. Building conditions can continue to deteriorate by the actions of earthquake

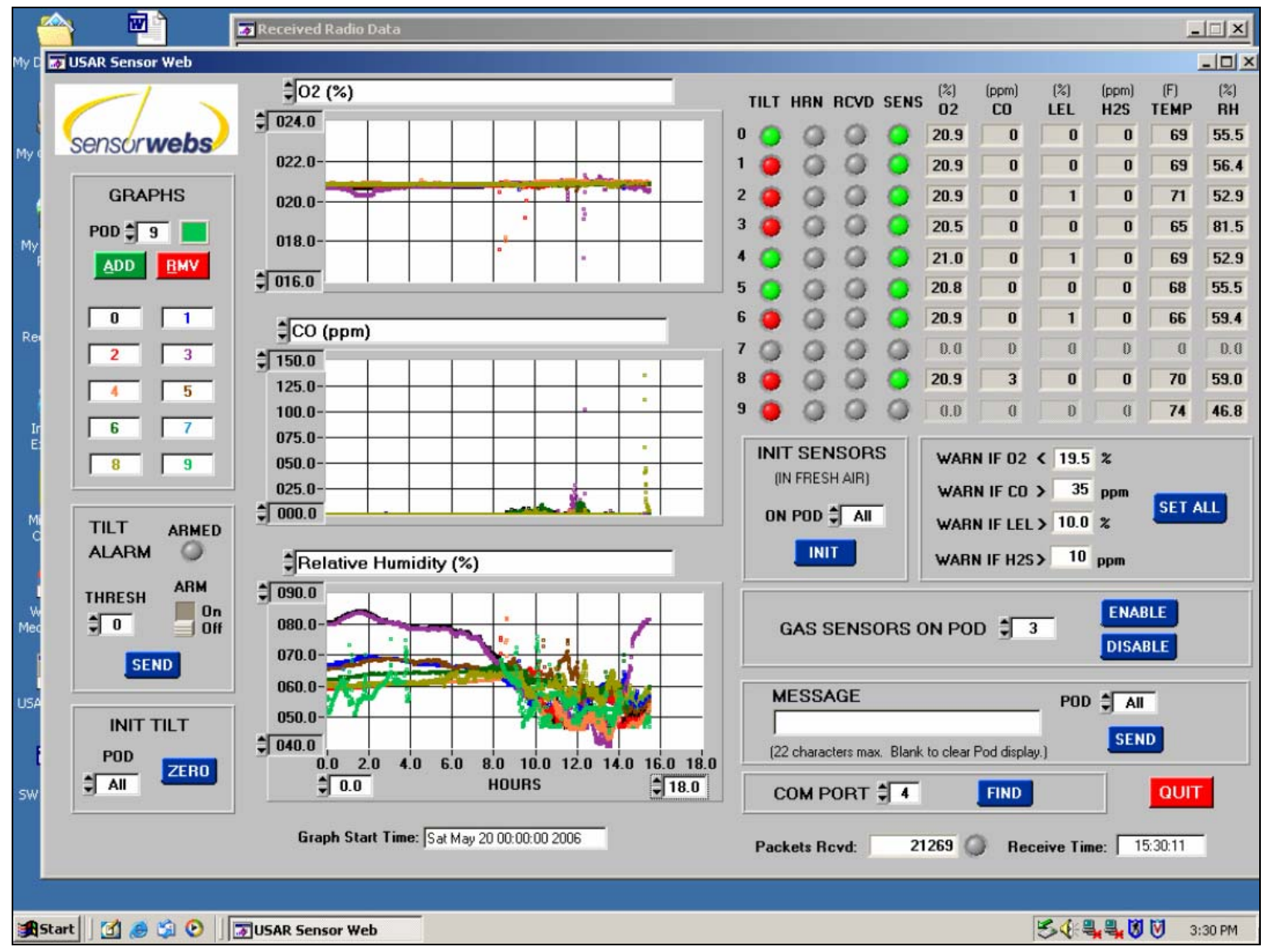

Figure 5: A screen-capture of the user interface for the Sensor Web. Time-based trending data are immediately available as are current readings from the system. The interface also serves as a command portal into the system. Note the spike in CO concentration at hour 15. This was a result of an acetylene torch being ignited inside the collapsed structure. 
aftershocks, wind, rain and snow loading, and the intentional or unintentional actions of rescue workers.

The 4-gas Sensor Web pods described above also have an accelerometer built into them. The accelerometer functions as a tilt-meter to determine a change of state in the pod's orientation. The pod, attached to shoring or a building wall, can monitor changes while they are occurring and warn of impending failure. Such pods therefore perform double-duty, monitoring both atmosphere and structural integrity with the attendant reporting benefits for both parameters (see Fig. 6).

Shoring stress tests revealed that the Sensor Web provided warning 60 seconds (2 measurement cycles) before shoring failure. Such an advanced warning, distributed throughout the entire space of operations, would greatly reduce the risk of personnel being caught in a further collapse.

\subsection{Decontamination Monitoring}

During biological decontamination operations, a chemical agent is introduced at the proper temperature and humidity to destroy the intended pathogen. Such operations are typically very complex logistically and labor intensive. The target structure to be decontaminated is

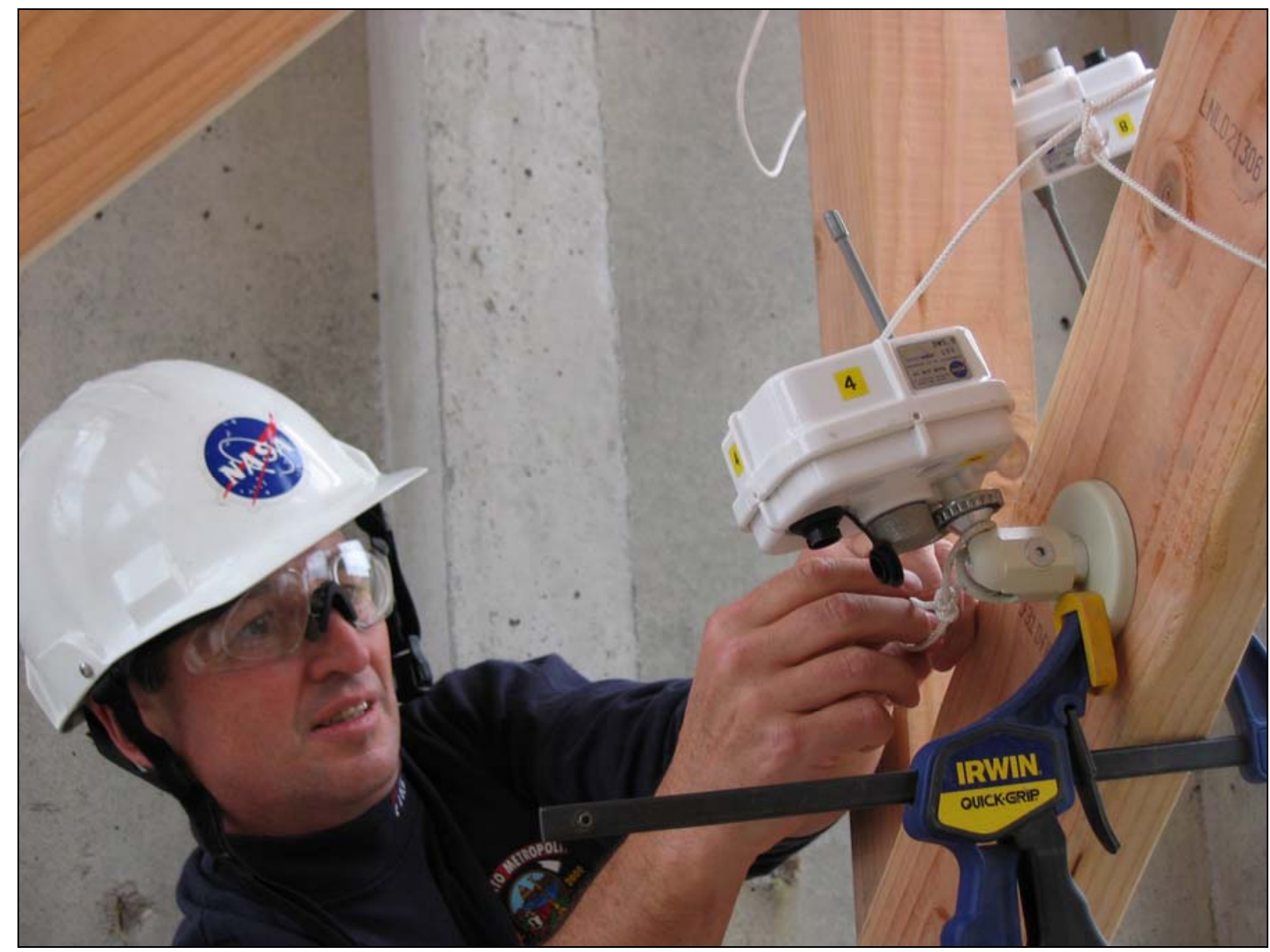

Figure 6: Pods are attached to shoring. 
first sealed with tarps that must hold the caustic decontaminant gas over many hours. Typical operation will have the caustic gas pumped into and out of the structure continually with small fans placed throughout the structure to ensure even distribution of the gas. The entire building's atmosphere needs to be monitored during the decontamination procedure to ensure that proper conditions exist (decontaminant concentration, temperature, and humidity) to kill the pathogen.

Presently, wet chemistry techniques are used to monitor the operation. Long plastic tubing is distributed throughout the structure from a central hub. Atmospheric samples are pumped out of the hub at regular intervals and examined chemically to maintain appropriate conditions in the building.

There are several disadvantages with this technique. First, the distribution of the plastic tubes can take nearly a full day, even in a modest-sized home. Second, sampling in this manner means that the chemical analysis will only yield the average conditions of the building due to gas mixing in the central hub. As a result, there still might be pockets in the structure where an appropriate concentration of decontaminant is not obtained, allowing for the pathogen to live. Lastly, water can collect in the plastic tubing due to the high humidity conditions typically needed during operations. This water can effectively absorb the gas $\left(\mathrm{ClO}_{2}\right.$ in the case of anthrax decontamination) which will create spurious results during chemical analysis. The tubes can also fill with ice during winter-time decontaminations and therefore make sample retrieval impossible and force operators to abort the operation entirely.

A Sensor Web with $\mathrm{ClO}_{2}$ sensors attached to each pod has been successfully used to alleviate these problems (see Fig. 7). Insertion of the technology into the operations was easy and personnel were able to learn the system in literally under 15 minutes. At least a dozen actual decontaminations have been performed using this system. In addition to the tremendous labor reduction in eliminating the tube system for gas sample retrieval, the Sensor Web allows operators to follow the $\mathrm{ClO}_{2}$ gas plume throughout the structures in real time. On more than one occasion, this enabled operators to immediately find leaks in the building's tarps as well as building pockets where $\mathrm{ClO}_{2}$ gas concentrations were lower than expected.

\section{FUTURE DIRECTIONS}

The Sensor Web is a general information infrastructure for sensors. It collects, analyzes and reacts to whatever conditions are important to the end-user, and can be coupled with existing methods of obtaining the necessary data. Any low-bandwidth sensor can be attached to the Sensor Web and enhance the situational awareness properties of the system. Initial experiments using a Sensor Web to track in-field personnel during operations show promise. Such a capability would benefit locating fire fighters lost in buildings as well as provide an accurate count of personnel going into and out of a rescue operation area.

Other applications for the Sensor Web immediately present themselves, especially involving infrastructure protection. The properties of the Sensor Web make it ideal for sentinel 

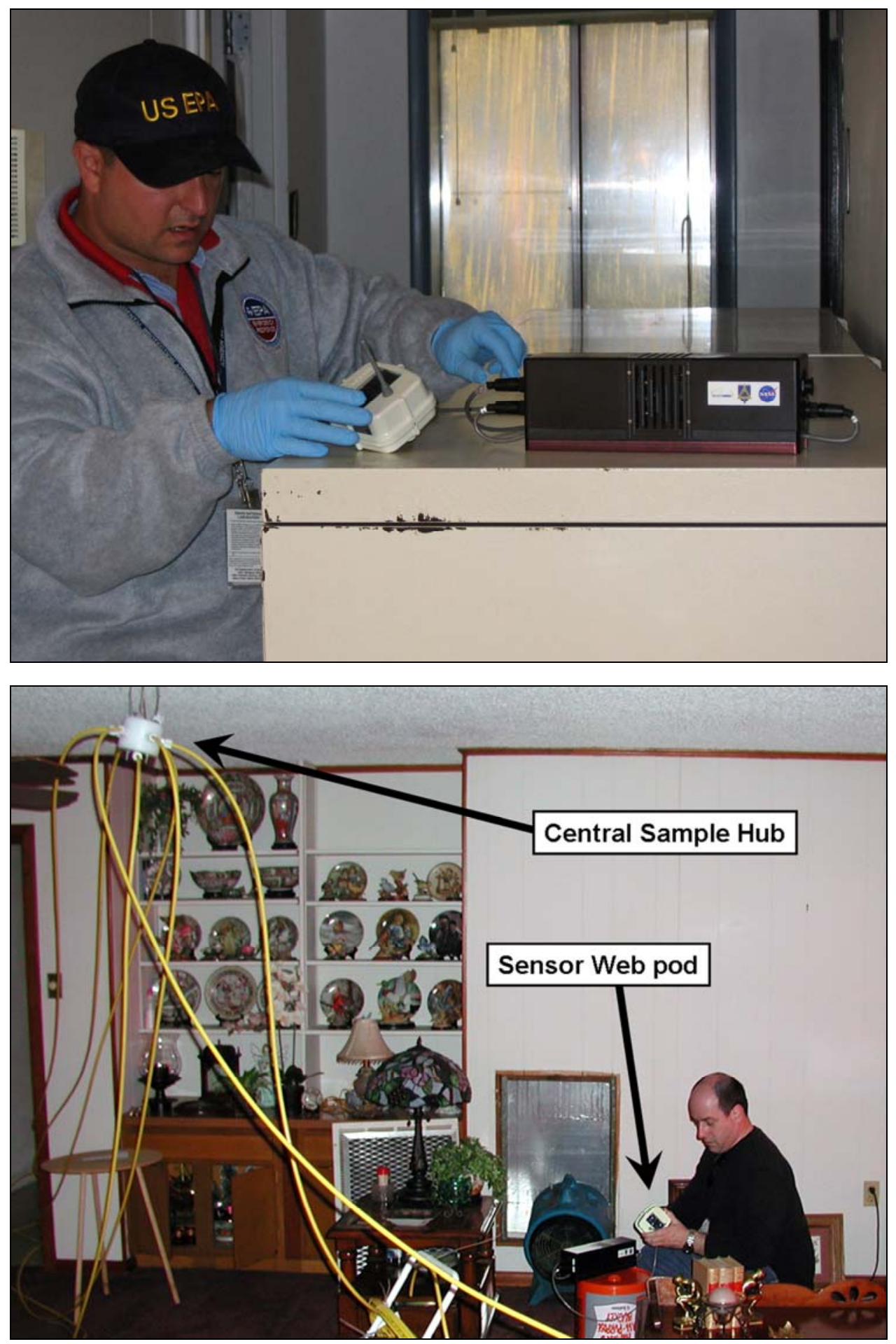

Figure 7: Sensor Web use for decontamination operations. Top: EPA decontamination team member deploying a Sensor Web pod. The black box attached to the pod is the $\mathrm{ClO}_{2}$ sensor. Bottom: A Sensor Web is deployed in a decontamination area that also uses the traditional sampling method to monitor $\mathrm{ClO}_{2}$ concentration. Note the traditional method requires plastic tubing that runs through the entire house and connects to a central hub where atmosphere samples will be pumped out for chemical analysis. 
security systems where a disturbance at one pod will be known by all. Moreover, the massively redundant connectivity of the macro-instrument makes the Sensor Web amenable to over-the-horizon monitoring of rail and highways.

Finally, the reactive capabilities of the Sensor Web to dynamic environments are only now being explored. Future systems may, for example, control ventilation during rescue operations based on changing atmospheric conditions and consequently free up additional labor that can be better applied to the actual rescue tasks at hand. Clearly, the situational awareness capability inherent in the Sensor Web can only increase with advances in the underlying technology and with no additional cost compared to that of more traditional, less capable wireless solutions.

\section{REFERENCES}

[1] Layman, Lloyd. Fire Fighting Tactics. 1953. National Fire Protection Association, Quincy, MA.

[2] Delin, K.A. The Sensor Web: A Macro-Instrument for Coordinated Sensing. Sensors. 2002; 2:270-285.

[3] Koch, C. and Laurent, G. Complexity and the Nervous System. Science. 1999; 284:9698.

[4] Delin, K.A. Sensor Webs in the Wild. pp. 259-272 in Bulusu, N. and S. Jha, S., eds. Wireless Sensor Networks: A Systems Perspective. 2005. Artech House, Norwood, MA.

[5] Delin, K.A., Jackson, S.P., Johnson, D.W., Burleigh, S.C., Woodrow, R.R., McAuley, J.M., Dohm, J.M., Ip, F., Ferré, T.P.A, Rucker, D.F., and Baker, V.R. Environmental Studies with the Sensor Web: Principles and Practice. Sensors. 2005; 5:103-117.

[6] See links at www.SensorWareSystems.com 